\title{
Douleur et addictions
}

\section{Pain and Addictions}

\section{A.-P. Trouvin $\cdot$ N. Authier}

(C) Lavoisier SAS 2019

Le contexte actuel, en Amérique du Nord notamment, « d'épidémie » de décès liés à la prise d'antalgiques dits de prescription amène aujourd'hui de nombreux pays européens dont la France à se pencher sur la question du mésusage et des effets indésirables graves liés à ces traitements.

Il convient tout d'abord de distinguer douleur aiguë, douleur chronique et douleur du cancer. Comme le rappelaient Jules Desmeules et Elisabeth Collin dans le numéro Douleur et Cancer du mois de mars, la prévalence de la douleur chez les patients atteints de cancer reste élevée, avec une insuffisance de traitement retrouvée dans $63 \%$ des cas. Un juste équilibre doit être trouvé entre prévention des risques et renforcement de la vigilance sur ces médicaments et le risque de revenir à une « opiophobie » de la part des professionnels de santé, qui nuirait à la bonne prise en charge des patients.

Concernant la douleur non cancéreuse, il est clair que l'usage des opioïdes reste source de débat quant à son efficacité dans la douleur chronique. Le très récent rapport de l'Agence nationale de sécurité du médicament et des produits de santé nous interpelle sur plusieurs aspects. Il existe d'une part une augmentation nette des prescriptions d'opioïdes forts

A.-P. Trouvin $(\square)$

Centre d'évaluation et traitement de la douleur,

hôpital Ambroise-Paré, 9, avenue Charles-de-Gaulle,

F-92100 Boulogne-Billancourt, France

e-mail : annepriscille.trouvin@aphp.fr

Inserm U987, hôpital Ambroise-Paré,

F-92100 Boulogne-Billancourt, France

\section{N. Authier}

Inserm 1107, service de pharmacologie médicale, structure douleur chronique,

centres addictovigilance et pharmacovigilance,

CHU Clermont-Ferrand, université Clermont-Auvergne,

BP69, F-63003 Clermont-Ferrand, France

Institut Analgesia, faculté de médecine, BP 38,

F-63001 Clermont-Ferrand, France

Inserm 1107, Observatoire français

des médicaments antalgiques (OFMA),

CHU de Clermont-Ferrand,

université Clermont-Auvergne, France mais aussi d'autre part une augmentation de la prescription chronique. À ces augmentations de prescriptions s'ajoute un retentissement sur les événements indésirables. En dix ans le taux de notification d'intoxication aux antalgiques opioïdes a presque doublé, et en 15 ans le nombre de décès liés à la consommation d'antalgiques opioïdes a plus que doublé.

Finalement, traiter la douleur, soulager le patient et éviter le mésusage et le risque d'addiction obligent à un équilibre difficile. Dans ce numéro, plusieurs articles nous rapportent les difficultés auxquelles peuvent être confrontés les prescripteurs. Dans le cas clinique rapporté par G. Chvetzoff, cette dualité est perceptible, une patiente nécessitant à un moment un traitement antalgique fort a néanmoins progressivement évolué vers un mésusage manifeste.

Ce mésusage, de plus en plus, ne cible pas uniquement les antalgiques opioïdes puisque, comme rapporté par G. Mick, $27 \%$ des patients recevant un traitement par prégabaline n'avaient pas une situation douloureuse pour laquelle cette molécule a une potentielle efficacité.

Face à tous ces signaux pour des molécules relativement « anciennes », quid de l'arrivée prochaine du cannabis thérapeutique ? Certains l'évoquent comme la solution de substitution aux opioïdes. Cependant, dans une large étude de cohorte (plus de 1500 patients douloureux chroniques traités par opioïdes), après quatre ans de suivi, seulement un quart des patients ont eu recours à du cannabis dans un but antalgique, et chez ces patients, aucun argument en faveur du cannabis n'a été retrouvé. Les patients qui consommaient du cannabis décrivaient une douleur plus intense, et rien n'indiquait que la consommation de cannabis a permis de diminuer le recours aux opioïdes. L'étude observationnelle d'O. Renard chez des patients ayant un cancer bronchique montre qu'effectivement la consommation de cannabis n'a pas d'impact sur la consommation d'opioïdes forts si ce n'est que dans le groupe de patients usagers de cannabis l'introduction d'opioïdes forts était significativement plus précoce que dans le groupe témoin. D'autres l'évoquent comme une ligne de traitement qui pourrait s'envisager avant les opioïdes, mais là aussi, à ce jour les preuves manquent sur l'efficacité du cannabis thérapeutique dans les douleurs chroniques non cancéreuses et non neuropathiques. 